\title{
Penetrating injuries to the vertebral artery: interventions and outcomes from US Trauma Centers
}

\author{
Morgan Schellenberg ${ }^{1} \cdot$ Natthida Owattanapanich $^{1} \cdot$ Shaun Cowan $^{1} \cdot$ Matt Strickland $^{1} \cdot$ Meghan Lewis $^{1}$. \\ Damon H. Clark ${ }^{1} \cdot$ Kenji Inaba ${ }^{1}$
}

Received: 15 April 2020 / Accepted: 12 June 2020 / Published online: 21 June 2020

○) Springer-Verlag GmbH Germany, part of Springer Nature 2020

\begin{abstract}
Purpose Penetrating injuries to the vertebral artery are rare and incompletely studied. Operative, angioembolic, and nonoperative strategies are management options, although the association between management strategy and outcomes is unknown. This study endeavored to define the epidemiology, management strategy, and outcomes after penetrating injuries to the vertebral artery presenting to trauma centers nationwide.

Methods Patients with veterbral artery injuries were identified from the National Trauma Data Bank (NTDB) (2016-2017) using ICD-10-CM codes. Only those with penetrating mechanisms of injury were included in the study. Transferred patients were excluded. Study groups were defined by management strategy (Operative management, OM; angioembolization, AE; and nonoperative management, NOM). Patient demographics, injury characteristics, and outcomes were compared between groups using univariate analysis. Multivariate analysis with logistic regression was used to examine independent risk factors for mortality and stroke.

Results Penetrating injuries to the vertebral artery were rare $(n=476,<1 \%$ of NTDB patient population). Median age was 28 [IQR 21-37] years and $81 \%(n=385)$ of patients were male. Interpersonal violence was the most common injury intent $(n=374,79 \%)$. Most patients were managed with NOM $(n=409,86 \%)$, with AE and OM utilized less frequently $(8 \%$ and $6 \%$, respectively). Stab wounds were the most frequent mechanism of injury among patients managed with OM (62\%), while gunshot wounds were most common among patients managed with NOM (84\%) or AE (79\%). Multivariate analysis of risk factors for stroke revealed only associated carotid artery injury (OR 4.236, 95\% CI 1.284-13.970, $p=0.018$ ) and AE (OR $6.342,95 \%$ CI $1.417-28.399, p=0.016)$ were independent predictors. Independent risk factors for mortality were advanced age (OR 1.026, 95\% CI 1.001-1.052, $p=0.044$ ); elevated ISS (OR 1.030, 95\% CI 1.008-1.052, $p=0.006$ ); and associated traumatic brain injury (OR 3.020, 95\% CI 1.333-6.843, $p=0.008$ ). Higher ED GCS was independently associated with reduced mortality (OR $0.788,95 \%$ CI $0.731-0.849, p<0.001)$.

Conclusions Vertebral artery injuries after penetrating mechanisms are infrequent in the United States. Patients with these injuries tend to be young adult men who were injured by gunshot wounds as a result of interpersonal violence. The majority of these injuries were managed nonoperatively, with operative intervention required most commonly for patients injured by stab wounds. Risk factors for both stroke and mortality were principally due to patient factors and associated injuries. Increased risk of stroke among patients managed with angioembolization will need to be further investigated with future study to determine if this risk is imparted from the management strategy itself or from underlying injury characteristics.
\end{abstract}

Keywords Vertebral artery injury $\cdot$ Penetrating trauma $\cdot$ Stroke $\cdot$ Interpersonal violence $\cdot$ Nonoperative management of penetrating trauma

Morgan Schellenberg

morgan.schellenberg@med.usc.edu

1 Division of Trauma and Surgical Critical Care, LAC + USC Medical Center, University of Southern California, 2051 Marengo Street, Inpatient Tower, C5L100, Los Angeles, CA 90033, USA

\section{Background}

Existing literature on penetrating injuries to the vertebral artery is comprised primarily of single-center case series $[1-8]$ and case reports, with a recent systematic review that offers a comprehensive summary of these study results [9]. 
Penetrating vertebral artery injuries are uncommon, with busy urban trauma centers encountering such patients in low single-digit numbers annually $[6,7]$. Traditional management options included nonoperative management as well as surgical ligation or, much less frequently, arterial repair. Vertebral artery injuries can be extremely challenging to expose operatively and the evolution of angioembolization as a treatment modality for these injuries has allowed for nonsurgical hemorrhage control. Previously, angioembolization was a less reliable treatment option for hemorrhage control or management of pseudoaneurysm or arteriovenous fistula after penetrating vertebral artery injuries, with success rates of approximately $60 \%$ [2]. As use and familiarity with the technique increased over time, a more recent study demonstrated a success rate approaching $90 \%$ [1]. The current literature does not delineate the differences in outcomes according to management strategy, which are of interest to any clinician who manages trauma patients. Furthermore, data on national-level epidemiology, interventions, and outcomes after penetrating injuries to the vertebral artery are lacking.

The primary study objective is to utilize a large, nationwide trauma database to examine the epidemiology, associated injuries, interventions, and outcomes of patients with injuries to the vertebral artery after penetrating trauma across the United States. Our hypothesis is that operative intervention will infrequently be required, and that stroke and mortality rates will be low among patients arriving alive to the hospital.

\section{Methods}

This retrospective observational study was performed with the National Trauma Data Bank (NTDB), which is the largest accumulation of US trauma center data and is led by the American College of Surgeons Committee on Trauma [10]. All patients from January 2016 to December 2017 who sustained a vertebral artery injury, as defined by the 10th revision of the International Statistical Classification of Diseases and Related Health Problems (ICD-10), were included. The only exclusion criterion was transfer from an outside hospital. Institutional Review Board approval was sought from the University of Southern California and the exemption was granted. Permission for waived consent was obtained given the retrospective observational study design and the de-identified nature of the NTDB dataset.

The variables examined included patient demographics and comorbidities (age, gender, race/ethnicity; and pre-existing diabetes mellitus [DM], hypertension [HTN], or stroke); injury data (year of injury, mechanism of injury, associated head/neck injuries, Abbreviated Injury Scale [AIS] score by body region, Injury Severity Score [ISS], initial emergency department [ED] vital signs and Glasgow Coma Scale [GCS] score); and outcomes (the need for surgical or angioembolic intervention, hospital length of stay [LOS], intensive care unit [ICU] LOS, ventilator days, complications, mortality, and discharge disposition). After extracting race and ethnicity from the NTDB, these variables were combined into a single variable with any patient of Hispanic ethnicity, regardless of race, coded as Hispanic.

Study groups were defined by intervention: operative management $(\mathrm{OM})$ vs. angioembolization (AE) vs. nonoperative management (NOM). Descriptive statistics were used to summarize the patient demographics, injury data, clinical data and outcomes. Continuous variables are presented as median (interquartile range, [IQR]) and categorical variables as numbers (percentages). To analyze the difference between the groups on a univariate level, the Chi-square $\left(\chi^{2}\right)$ test was used for categorical variables and Kruskal-Wallis rank-sum test was used for continuous data.

Multivariate logistic regression was performed to identify independent risk factors for stroke. Variables with $p<0.2$ on univariate analysis were included. The model fit was assessed using the Hosmer-Lemeshow test. Results of multivariate analysis are expressed as odds ratios (OR) with $95 \%$ confidence intervals (CI). Statistical significance was defined as $p<0.05$. All statistical analyses were performed using IBM SPSS Statistics 23 (IBM Corporation; Armonk, NY).

\section{Results}

\section{Patient demographics}

Over the study period, vertebral artery injuries comprised $<1 \%(n=6865)$ of the total NTDB patient population ( $n=1,965,144)$ (Fig. 1). Of these, only $9 \%$ occurred after penetrating trauma. After exclusions, 476 patients remained for analysis. Median age was 28 [IQR 21-37] years and $81 \%(n=385)$ of patients were male (Table 1$)$. No patient in this study had a prior history of stroke. The incidences of DM and HTN were low and did not vary by study group (Table 1).

\section{Clinical and injury data}

Patients with penetrating vertebral artery injuries frequently arrived to the ED comatose $(n=161,34 \%)$ (Table 1). Hypotension or tachycardia on arrival was less frequent $(20 \%$ and $19 \%$, respectively). Interpersonal violence was the most common intent of injury $(n=374,79 \%)$ and gunshot wounds were the most frequent mechanism $(n=383,80 \%)$. Median ISS was 18 [IQR 12-29].

Associated injuries to the head and neck were infrequent and are listed in Table 1. Patients managed with OM were 


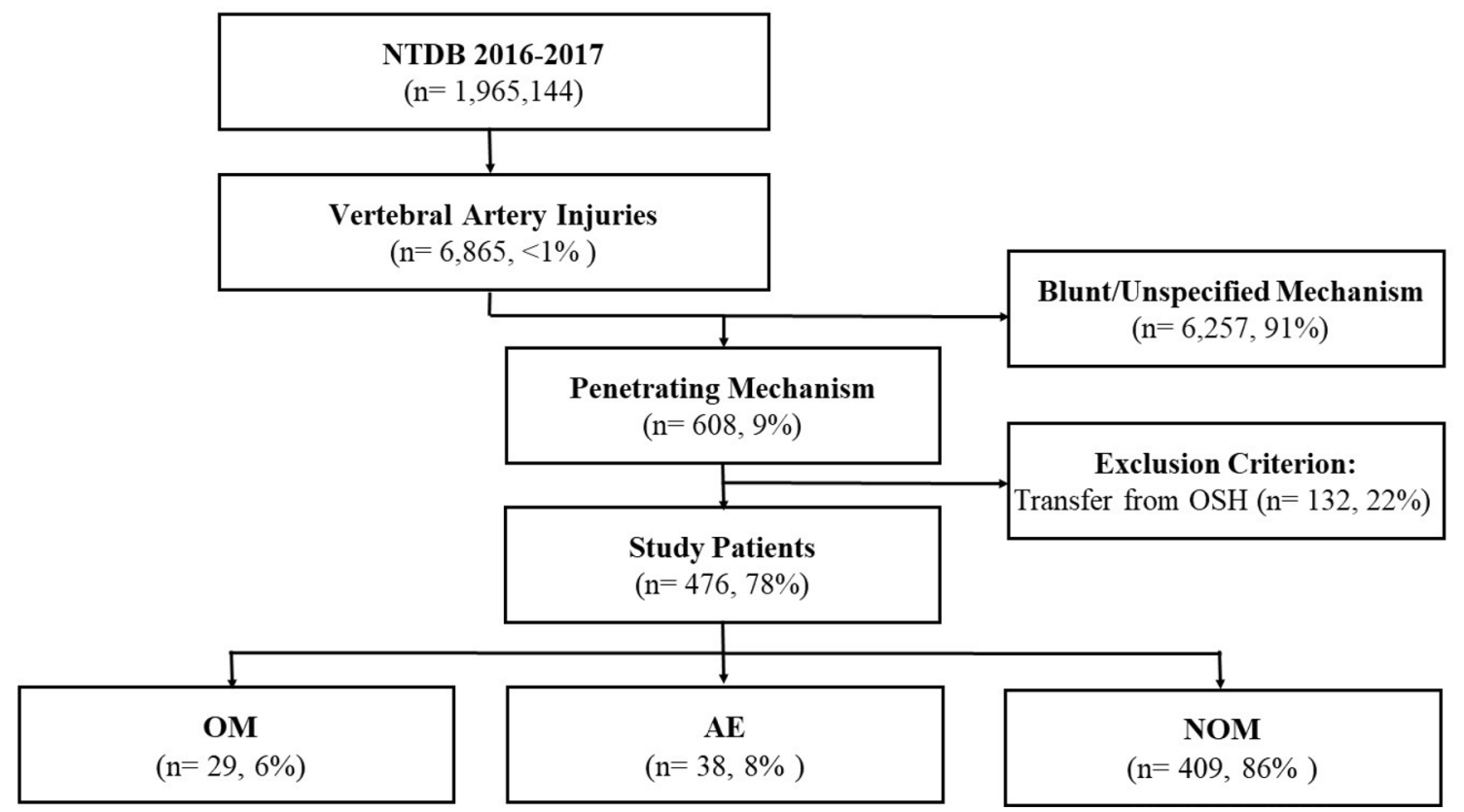

Fig. 1 Flow of patients through the study. $N T D B$ National Trauma Data Bank, $O S H$ outside the hospital, $O M$ operative management, $A E$ angioembolization, NOM nonoperative management

more likely to have an associated injury to the internal or external jugular vein ( $41 \%$ vs. $8 \%$ vs. $8 \%, p<0.001)$ but less likely to have an associated cervical spine fracture (24\% vs. $66 \%$ vs. $62 \%, p<0.001)$.

\section{Management strategy}

Most patients were managed with NOM $(n=409,86 \%)$, with $\mathrm{AE}$ and $\mathrm{OM}$ utilized less frequently (8\% and 6\%, respectively) (Table 1). Management strategy varied by study year (Fig. 2). Stab wounds were the most frequent mechanism of injury among patients managed with OM (62\%), while gunshot wounds were most common among patients managed with NOM $(84 \%)$ or AE (79\%) (Table 1) (Fig. 3). Time to the intervention was shorter among patients managed with OM than AE (1 [1-3] vs. 5 [3-14] hours, $p<0.001)$ (Table 2). Complications did not differ according to management strategy apart from the risk of stroke. Stroke occurred most frequently among OM patients $(10 \%)$, followed by AE $(8 \%)$ and NOM $(2 \%)$ patients $(p=0.020)$.

\section{Outcomes}

Univariate analysis of outcomes is provided in Table 2. The overall risk of stroke after a penetrating vertebral artery injury was 3\% (Table 2). Multivariate analysis of independent risk factors for stroke revealed only associated carotid artery injury (OR 4.236, 95\% CI 1.284-13.970, $p=0.018$ ) and AE (OR 6.342, 95\% CI 1.417-28.399, $p=0.016)$ to be independently associated with in-hospital stroke risk (Table 3).

Overall mortality after penetrating vertebral artery injury was $19 \%$ (Table 2). On multivariate analysis, mortality was independently associated with increased age (OR 1.026, 95\% CI 1.001-1.052, $p=0.044$ ); elevated ISS (OR 1.030, 95\% CI 1.008-1.052, $p=0.006)$; and associated traumatic brain injury (OR 3.020, 95\% CI 1.333-6.843, $p=0.008$ ) (Table 4). Higher ED GCS was independently associated with reduced mortality (OR 0.788, 95\% CI 0.731-0.849, $p<0.001$ ).

\section{Discussion}

Injuries to the vertebral artery are much more commonly blunt than penetrating. The rarity of penetrating vertebral artery injuries limits our understanding of the demographics of the patients who are affected, the frequency of need for surgical intervention, and outcomes. Furthermore, the current literature [1-9] does not delineate the impact of management strategy on outcomes after injury, particularly in terms of stroke and mortality. The current study was undertaken to answer these questions, utilizing a large nationwide study population of trauma patients across the United States.

This study revealed that the demographics of patients with injured vertebral arteries are consistent with those of patients most at risk for penetrating injury in general: men in the third and fourth decades of life. Tachycardia and hypotension on presentation were infrequent, which is 
Table 1 Patient demographics, clinical data, and injury data

\begin{tabular}{|c|c|c|c|c|c|}
\hline & Total $(n=476)$ & $\mathrm{OM}(n=29,6 \%)$ & $\mathrm{AE}(n=38,8 \%)$ & $\operatorname{NOM}(n=409,86 \%)$ & $p$ \\
\hline \multicolumn{6}{|l|}{ Demographic data } \\
\hline Age, years & 28 [21-37] & $32[23-53]$ & 28 [20-36] & 28 [21-36] & 0.267 \\
\hline Gender, male & $385(81 \%)$ & $24(83 \%)$ & $33(87 \%)$ & $328(80 \%)$ & 0.588 \\
\hline Race & & & & & 0.974 \\
\hline Black & $228(48 \%)$ & $12(41 \%)$ & $20(52 \%)$ & $196(48 \%)$ & \\
\hline White & $171(36 \%)$ & $10(35 \%)$ & $14(37 \%)$ & $147(36 \%)$ & \\
\hline Hispanic & $37(8 \%)$ & $3(10 \%)$ & $1(3 \%)$ & $33(8 \%)$ & \\
\hline Other & $40(8 \%)$ & $4(14 \%)$ & $3(8 \%)$ & $33(8 \%)$ & \\
\hline \multicolumn{6}{|l|}{ Comorbidities } \\
\hline $\mathrm{DM}$ & $4(1 \%)$ & $1(3 \%)$ & $1(3 \%)$ & $2(1 \%)$ & 0.109 \\
\hline HTN & $7(2 \%)$ & $0(0 \%)$ & $1(3 \%)$ & $6(2 \%)$ & 0.675 \\
\hline \multicolumn{6}{|l|}{ Clinical data } \\
\hline HR & 93 [75-113] & 105 [92-129] & 105 [80-124] & $91[74-110]$ & 0.001 \\
\hline $\mathrm{HR}>120$ & $86(19 \%)$ & $9(31 \%)$ & $11(29 \%)$ & $66(17 \%)$ & 0.033 \\
\hline SBP & 117 [97-140] & 106 [92-128] & 121 [102-140] & 117 [97-140] & 0.115 \\
\hline $\mathrm{SBP}<90$ & $92(20 \%)$ & $6(22 \%)$ & $6(16 \%)$ & $80(20 \%)$ & 0.815 \\
\hline GCS & $14[3-15]$ & $14[3-15]$ & $15[3-15]$ & $14[3-15]$ & 0.759 \\
\hline GCS $<9$ & $161(34 \%)$ & $12(41 \%)$ & $12(32 \%)$ & $137(34 \%)$ & 0.681 \\
\hline \multicolumn{6}{|l|}{ Injury data } \\
\hline Intent & & & & & 0.368 \\
\hline Interpersonal violence & $374(79 \%)$ & $20(69 \%)$ & $30(79 \%)$ & $324(79 \%)$ & \\
\hline Self-inflicted & $42(9 \%)$ & $6(21 \%)$ & $5(13 \%)$ & $31(8 \%)$ & \\
\hline Accidental & $33(7 \%)$ & $2(7 \%)$ & $1(3 \%)$ & $30(7 \%)$ & \\
\hline Undetermined & $27(5 \%)$ & $1(3 \%)$ & $2(5 \%)$ & $24(6 \%)$ & \\
\hline Mechanism & & & & & $<0.001$ \\
\hline Gunshot wound & $383(80 \%)$ & $11(38 \%)$ & $30(79 \%)$ & $342(84 \%)$ & \\
\hline Stab wound & $90(19 \%)$ & $18(62 \%)$ & $8(21 \%)$ & $64(15 \%)$ & \\
\hline Other penetrating & $3(1 \%)$ & $0(0 \%)$ & $0(0 \%)$ & $3(1 \%)$ & \\
\hline ISS & 18 [12-29] & $18[11-26]$ & 17 [11-25] & 20 [13-29] & 0.159 \\
\hline \multicolumn{6}{|l|}{ Associated injuries } \\
\hline TBI & $58(12 \%)$ & $0(0 \%)$ & $6(16 \%)$ & $52(13 \%)$ & 0.101 \\
\hline Pharynx & $30(6 \%)$ & $1(3 \%)$ & $3(8 \%)$ & $26(6 \%)$ & 0.754 \\
\hline Larynx & $26(6 \%)$ & $1(3 \%)$ & $4(11 \%)$ & $21(5 \%)$ & 0.333 \\
\hline Trachea & $10(2 \%)$ & $0(0 \%)$ & $0(0 \%)$ & $10(2 \%)$ & 0.433 \\
\hline Esophagus & $16(3 \%)$ & $2(7 \%)$ & $1(3 \%)$ & $13(3 \%)$ & 0.543 \\
\hline Carotid artery & $84(18 \%)$ & $7(24 \%)$ & $3(8 \%)$ & $74(18 \%)$ & 0.184 \\
\hline Internal or external JV & $48(10 \%)$ & $12(41 \%)$ & $3(8 \%)$ & $33(8 \%)$ & $<0.001$ \\
\hline Cervical vertebral column & $284(60 \%)$ & $7(24 \%)$ & $25(66 \%)$ & $252(62 \%)$ & $<0.001$ \\
\hline Cervical spinal cord & $100(21 \%)$ & $3(10 \%)$ & $7(18 \%)$ & $90(22 \%)$ & 0.303 \\
\hline
\end{tabular}

Continuous variables presented as median [interquartile range]. Categorical variables presented as number (\%)

$O M$ operative management, $A E$ management with angioembolization, $N O M$ nonoperative management, $D M$ diabetes mellitus, $H T N$ hypertension, $H R$ heart rate (beats per minute), SBP systolic blood pressure (mmHg), GCS Glasgow Coma Scale score, ISS Injury Severity Score, TBI traumatic brain injury (epidural hematoma, subdural hematoma, and/or subarachnoid hemorrhage). Carotid artery, includes common, internal, and external carotid arteries. $J V$ jugular vein

noteworthy as hemodynamic instability in these patients mandates a very challenging surgical exploration. Interpersonal violence with firearms was the most common intent and mechanism of injury, respectively, highlighting the need for public health efforts directed towards violence prevention to decrease the incidence of injury.

Interestingly, most patients who underwent surgical intervention were injured by stab wounds, despite stab wounds 


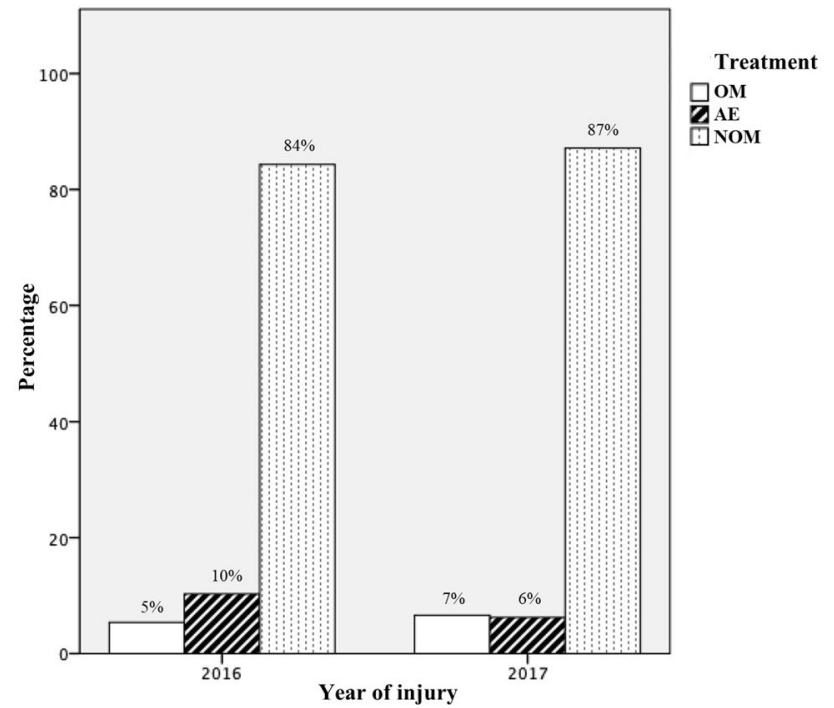

Fig. 2 Treatment of penetrating vertebral artery injuries by study year. Percentages are given as a number of patients managed with each treatment type divided by the total number of patients in each study year. $O M$ operative management, $A E$ angioembolization, $N O M$ nonoperative management

comprising the minority of vertebral artery injuries overall. Although there are several plausible explanations, the most likely seems that patients who are exsanguinating from a vertebral artery injury are most likely to survive to the hospital if the wounding mechanism is a knife. Although data from the Coroner's office were not captured by this study, the implication of gunshot wounds comprising the majority of cases but the minority of those being taken to the operating room is that patients with hemorrhage from gunshot wounds to the vertebral artery either sustain a minor injury or die before they reach the hospital. This suggests the importance of prehospital hemorrhage control to improve survival after vertebral artery injuries.

In-hospital stroke was relatively uncommon, but after adjusting for potential confounders, the risk of stroke was independently associated only with associated carotid artery injury or angioembolization. It is unclear if the increased risk of stroke after angioembolization is a result of the intervention itself or a reflection of injury characteristics among patients requiring intervention. This will require further investigation in the future.

Both the complexity of management as well as the rarity of these injuries are due in part to the relatively protected anatomic course of the vertebral artery. The first portion, V1, is the first cephalad branch off of the subclavian artery [11]. The vertebral artery in this location is relatively accessible once surgical exposure is achieved, particularly when compared to the second or third portion of the vertebral artery, $\mathrm{V} 2$ and V3. The V2 segment commences as V1 enters the transverse foramen of the sixth cervical vertebra. Surgical

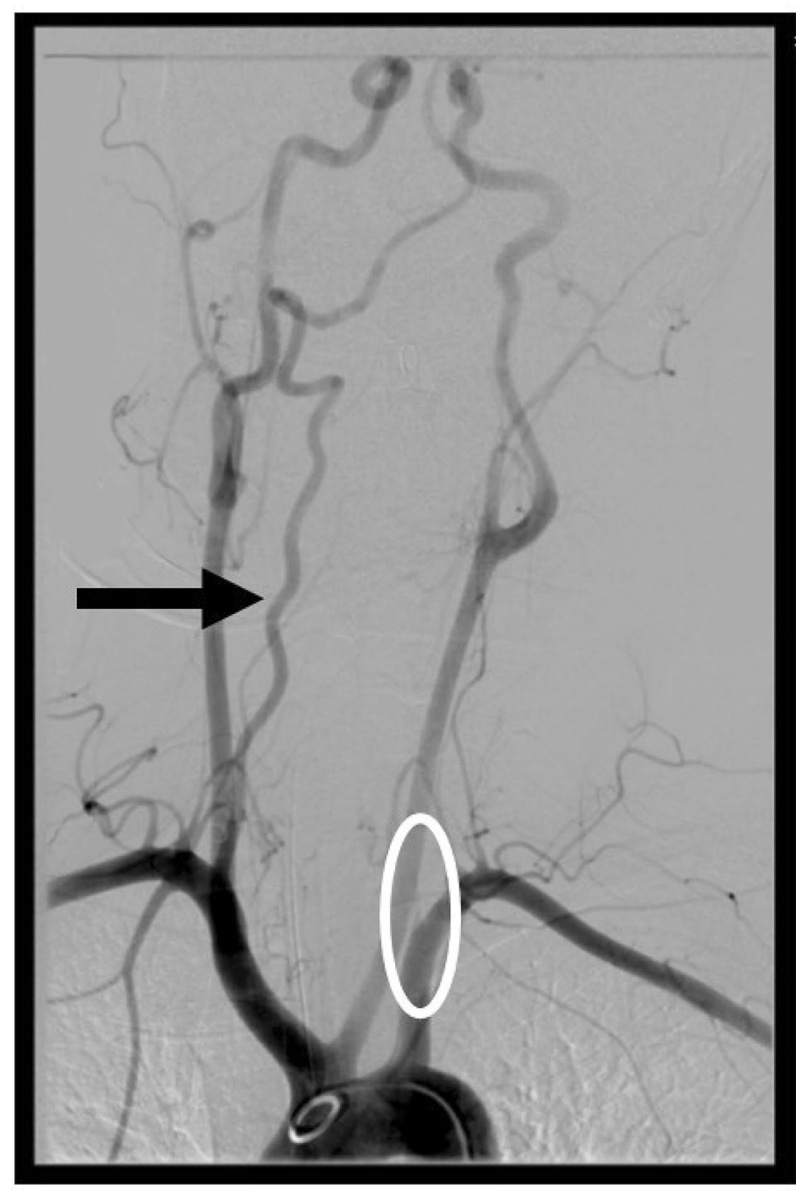

Fig. 3 Angiography for penetrating vertebral artery injury. A gunshot wound to the first portion of the left vertebral artery (circle) results in flow occlusion without retrograde filling as seen on catheter-based angiography. The contralateral (right) vertebral artery can be seen opacified (arrow) for comparison. Incidental note is made of a bovine aortic arch

exposure of $\mathrm{V} 2$ requires segmental osteotomies of the transverse processes of C1-C6 (Fig. 4), maneuvers which can be particularly challenging when the surgical field is obscured by ongoing hemorrhage. Injuries to $\mathrm{V} 3$, from the transverse foramen of $\mathrm{C} 1$ to the skull base, are also challenging to manage, especially because distal vascular control is hindered by the cranial base. For these reasons, surgical intervention for penetrating vertebral artery injuries is reserved for patients who are exsanguinating.

The limitations of the study must be considered in the interpretation of the results. First, there is a lack of data granularity imparted from registry-based study, and many components of vertebral artery injury assessment and management are left unanswered by this study. For example, diagnostic work-up for hemodynamically normal patients would be of interested but cannot be accurately gleaned from the NTDB. The quantification of blood loss by transfusion volume may have improved our understanding of 
Fig. 4 Operative management for penetrating vertebral artery injury. A stab wound to the second portion of the left vertebral artery (arrow) is exposed after segmental osteotomies of the transverse processes of the cervical vertebrae

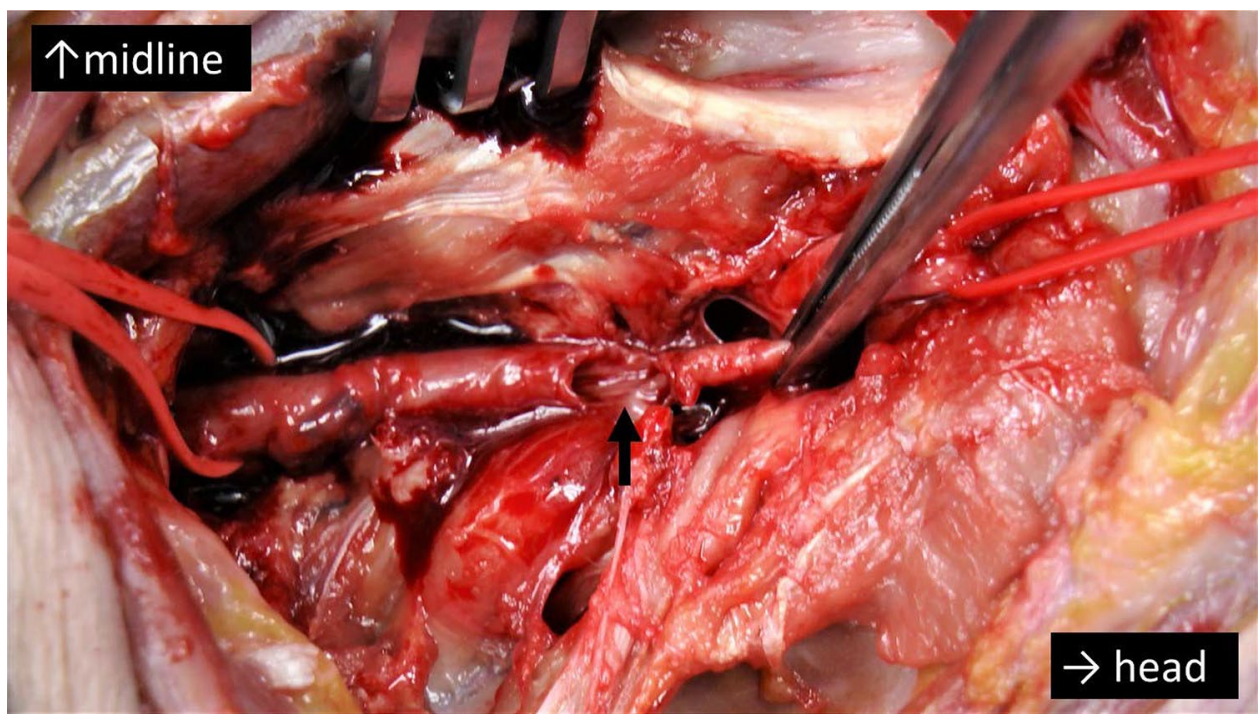

Table 2 Outcomes after penetrating vertebral artery injuries

\begin{tabular}{|c|c|c|c|c|c|}
\hline & Total $(n=476)$ & $\mathrm{OM}(n=29,6 \%)$ & $\mathrm{AE}(n=38,8 \%)$ & $\operatorname{NOM}(n=409,86 \%)$ & $p$ \\
\hline Time to intervention, hours & - & $1[1-3]$ & $5[3-14]$ & - & $<0.001$ \\
\hline Mortality & $89(19 \%)$ & $8(28 \%)$ & $2(5 \%)$ & $79(19 \%)$ & 0.047 \\
\hline Hospital LOS, days & $12[6-23]$ & $10[5-10]$ & $16[9-19]$ & $11[6-23]$ & 0.179 \\
\hline ICU LOS, days & $6[3-14]$ & 5 [3-9] & $10[4-15]$ & $6[3-14]$ & 0.401 \\
\hline Ventilator days & $4[2-10]$ & $3[2-6]$ & $5[2-12]$ & $4[2-12]$ & 0.124 \\
\hline \multicolumn{6}{|l|}{ Complications } \\
\hline Cardiac arrest & $37(8 \%)$ & $2(7 \%)$ & $1(3 \%)$ & $34(8 \%)$ & 0.450 \\
\hline Pneumonia & $33(7 \%)$ & $1(3 \%)$ & $2(5 \%)$ & $30(7 \%)$ & 0.666 \\
\hline VTE & $21(4 \%)$ & $3(10 \%)$ & $0(0 \%)$ & $18(4 \%)$ & 0.124 \\
\hline Stroke & $16(3 \%)$ & $3(10 \%)$ & $3(8 \%)$ & $10(2 \%)$ & 0.020 \\
\hline Unplanned OR & $17(4 \%)$ & $2(7 \%)$ & $0(0 \%)$ & $15(4 \%)$ & 0.309 \\
\hline Unplanned intubation & $13(3 \%)$ & $1(3 \%)$ & $3(8 \%)$ & $9(2 \%)$ & 0.116 \\
\hline Unplanned readmission to ICU & $8(2 \%)$ & $3(10 \%)$ & $0(0 \%)$ & $5(1 \%)$ & 0.001 \\
\hline AKI & $8(2 \%)$ & $1(3 \%)$ & $0(0 \%)$ & $7(2 \%)$ & 0.549 \\
\hline Discharge disposition & & & & & 0.038 \\
\hline Home & $198(51 \%)$ & $13(62 \%)$ & $12(33 \%)$ & $173(52 \%)$ & \\
\hline Rehabilitation & $40(10 \%)$ & $1(5 \%)$ & $5(14 \%)$ & $34(10 \%)$ & \\
\hline Transfer to OSH & $72(19 \%)$ & $1(5 \%)$ & $10(28 \%)$ & $61(18 \%)$ & \\
\hline LTAC & $14(4 \%)$ & $0(0 \%)$ & $3(8 \%)$ & $11(4 \%)$ & \\
\hline Psychiatric hospital & $11(3 \%)$ & $2(9 \%)$ & $1(3 \%)$ & $8(3 \%)$ & \\
\hline SNF & $11(3 \%)$ & $2(9 \%)$ & $2(6 \%)$ & $7(2 \%)$ & \\
\hline AMA & $7(2 \%)$ & $1(5 \%)$ & $0(0 \%)$ & $6(2 \%)$ & \\
\hline Other & $34(8 \%)$ & $1(5 \%)$ & $3(8 \%)$ & $30(9 \%)$ & \\
\hline
\end{tabular}

Continuous variables presented as median (interquartile range). Categorical variables presented as number (\%)

$O M$ operative management, $A E$ management with angioembolization, NOM nonoperative management, $L O S$ length of stay, ICU intensive care unit, $V T E$ venous thromboembolism, $O R$ operation, $A K I$ acute kidney injury, $O S H$ outside hospital, $S N F$ skilled nursing facility, $A M A$ patient left against medical advice, LTAC long term acute care facility

stroke risk, but this information is not captured in the NTDB. Data on the use of antithrombotic or antiplatelet therapy after penetrating vertebral artery injuries may be helpful but are not coded within the NTDB. Second, retrospective observational studies are inherently unable to offer causal explanations for studied associations. Lastly, 
Table 3 Multivariate analysis of independent risk factors for stroke

\begin{tabular}{|c|c|c|c|}
\hline & \multicolumn{3}{|l|}{ Stroke } \\
\hline & Adj $p$ value & OR & $95 \% \mathrm{CI}$ \\
\hline Age & 0.993 & 1.000 & $(0.968-1.033)$ \\
\hline Gender, male & 0.353 & 0.512 & $(0.125-2.104)$ \\
\hline ED SBP $<90$ & 0.679 & 0.743 & $(0.181-3.041)$ \\
\hline ED GCS & 0.263 & 0.931 & $(0.821-1.055)$ \\
\hline ISS & 0.078 & 1.028 & $(0.997-.061)$ \\
\hline \multicolumn{4}{|l|}{ Mechanism } \\
\hline Gunshot wound & REF & & \\
\hline Stab wound & 0.896 & 0.893 & $(0.165-4.847)$ \\
\hline \multicolumn{4}{|l|}{ Asssociated injuries } \\
\hline Carotid artery & 0.018 & 4.236 & $(1.284-13.970)$ \\
\hline Internal or external JV & 0.777 & 1.242 & $(0.278-5.552)$ \\
\hline $\mathrm{OM}$ & 0.111 & 4.529 & $(0.708-28.962)$ \\
\hline $\mathrm{AE}$ & 0.016 & 6.342 & $(1.417-28.399)$ \\
\hline
\end{tabular}

Test for multicollinearity was performed prior to multivariate analysis. AUROC $=0.833(95 \% \mathrm{CI}=0.714-0.952)$

$O R$ odds ratio, $C I$ confidence interval, $E D$ emergency department, $S B P$ systolic blood pressure $(\mathrm{mmHg}), G C S$ Glasgow Coma Scale score, ISS Injury Severity Scale score. Carotid artery, includes common, internal, and external carotid arteries. $J V$ jugular vein, $O M$ operative management, $A E$ angioembolization

it is possible that the affected patient demographics vary geographically and, therefore, these results may not be appropriately extrapolated to other countries. These limitations could be addressed in the future with a prospective multicenter study.

In summary, this is the largest published study of patients with penetrating vertebral artery injuries, which are exceedingly rare. Affected patients tend to be young adult men and gunshot wounds were the most common mechanism of injury. The majority of these patients can be managed nonoperatively. When operative intervention is required, this typically occurs for patients injured by stab wounds. Injury prevention and impact minimization efforts for penetrating vertebral artery injuries could be directed towards interpersonal violence prevention and prehospital hemorrhage control.

Funding No funding was received for this study.

\section{Compliance with ethical standards}

Conflict of interest Authors Schellenberg, Owattanapanich, Cowan, Strickland, Lewis, Clark, and Inaba declare that they have no conflict of interest.

Ethical approval All procedures performed in this retrospective observational study involving human participants were in accordance with the ethical standards of the Institutional Review Board of the University of California (HS-20-00210) and with the 1964 Helsinki declaration and its later amendments or comparable ethical standards.
Table 4 Multivariate analysis of independent risk factors for mortality

\begin{tabular}{llll}
\hline & \multicolumn{2}{l}{ Mortality } & \\
\cline { 2 - 4 } & Adj $p$ value & OR & $95 \%$ CI \\
\hline Age & 0.044 & 1.026 & $(1.001-1.052)$ \\
Gender, male & 0.710 & 1.178 & $(0.497-2.791)$ \\
ED SBP $<90$ & 0.206 & 1.569 & $(0.781-3.150)$ \\
ED GCS & $<0.001$ & 0.788 & $(0.731-0.849)$ \\
ISS & 0.006 & 1.030 & $(1.008-1.052)$ \\
Mechanism of injury & & & \\
Gunshot wound & REF & & \\
Stab wound & 0.433 & 0.633 & $(0.201-1.987)$ \\
Asssociated injuries & & & \\
TBI & 0.008 & 3.020 & $(1.333-6.843)$ \\
Larynx & 0.133 & 0.275 & $(0.051-1.483)$ \\
Carotid artery & 0.869 & 0.934 & $(0.411-2.121)$ \\
Internal or external JV & 0.211 & 1.801 & $(0.717-4.528)$ \\
Cervical vertebral column & 0.863 & 0.939 & $(0.457-1.929)$ \\
Cervical spinal cord & 0.365 & 0.687 & $(0.305-1.548)$ \\
OM & 0.405 & 1.810 & $(0.448-7.320)$ \\
AE & 0.183 & 0.369 & $(0.085-1.598)$ \\
Stroke & 0.343 & 2.023 & $(0.471-8.690)$ \\
\hline
\end{tabular}

Test for multicollinearity was performed prior to multivariate analysis. AUROC $=0.902(95 \% \mathrm{CI}=0.864-0.939)$

$O R$ odds ratio, $C I$ confidence interval, $E D$ emergency department, $S B P$ systolic blood pressure $(\mathrm{mmHg})$, GCS Glasgow Coma Scale score, ISS Injury Severity Scale score, TBI traumatic brain injury (epidural hematoma, subdural hematoma, and/or subarachnoid hemorrhage). Carotid artery, includes common, internal, and external carotid arteries. $J V$ jugular vein, $O M$ operative management, $A E$ angioembolization

Informed consent This study received a waiver for informed consent by the Institutional Review Board of the University of Southern California (HS-20-00210).

\section{References}

1. Mwipatayi BP, Jeffery P, Beningfield SJ, Motale P, Tunnicliffe J, Navsaria PH. Management of extra-cranial vertebral artery injuries. Eur J Vasc Endovasc Surg. 2004;27:157-62.

2. Demetriades D, Theodorou D, Asensio J, Golshani S, Belzberg $\mathrm{H}$, Yellin A, Weaver F, Berne TV. Management options in vertebral artery injuries. Br J Surg. 1996;83(1):83-6.

3. Yee LF, Olcott EW, Knudson MM, Lim RC. Extraluminal, transluminal, and observational treatment for vertebral artery injuries. J Trauma. 1995;39:480-6.

4. Hatzitheofilou C, Demetriades D, Melissas J, Stewart M, Franklin J. Surgical Approaches to Vertebral Artery Injuries. Br J Surg. 1988;75:234-7.

5. Reid JD, Weigelt JA. Forty-three cases of vertebral artery trauma. J Trauma. 1988;28:1007-122. 
6. Blickenstaff KL, Weaver FA, Yellin AE, Stain SC, Finck E. Trends in the management of traumatic vertebral artery injuries. Am J Surg. 1989;158:101-6.

7. Golueke P, Sclafani S, Phillips T, Goldstein A, Scalea T, Duncan A. Vertebral artery injury-diagnosis and management. J Trauma. 1987;27:856-65.

8. Meier DE, Brink BE, Fry WJ. Vertebral artery trauma: acute recognition and treatment. Arch Surg. 1981;116:236-9.

9. Asensio JA, Dabestani PJ, Wenzl FA, Milijkovic SS, Kessler JJ, Fernandez CA, Becker T, Cornell D, Siu M, Voigt C, Agrawal
DK. A systematic review of penetrating extracranial vertebral artery injuries. J Vasc Surg. 2020. https://doi.org/10.1016/j. jvs.2019.10.084 [Epub ahead of print].

10. American College of Surgeons. National Trauma Data Bank: NTDB research data set admission years 2007-2014. Chicago, IL; 2017.

11. Demetriades D, Schellenberg M, Nash N. Vertebral artery. In: Demetriades D, Inaba K, Velmahos GC (Eds) Atlas of surgical techniques in trauma, 2nd Ed., 2019. 